International Mathematical Forum, 2, 2007, no. 27, 1311 - 1317

\title{
Symplectic Capacities of Classical Domains ${ }^{1}$
}

\author{
Guangcun $\mathrm{Lu}^{2}$ \\ Department of Mathematics \\ Beijing Normal University \\ Beijing 100875, P. R. China \\ gclu@bnu.edu.cn \\ Hao Ding \\ Department of Mathematics \\ Beijing Normal University \\ Beijing 100875, P. R. China \\ Qiao Zhang \\ Department of Mathematics and Physics \\ ZhengZhou Institute of Aeronautical Industry Management \\ ZhengZhou 450015, P. R. China
}

Abstract. In this note we calculate Gromov symplectic width and HoferZehnder symplectic capacity for the classical domains.

Mathematics Subject Classification: 53D35, 57R17, 53D05

Keywords: Gromov symplectic width, Hofer-Zehnder symplectic capacity, classical domains

\section{INTRODUCTION AND MAIN RESULTS}

Recall that the Gromov symplectic width of a symplectic manifold $(M, \omega)$ of dimension $2 n$ is defined by

$$
\mathcal{W}_{G}(M, \omega):=\sup \left\{\pi r^{2} \mid \exists \text { symplectic embedding }\left(B^{2 n}(r), \omega_{0}\right) \rightarrow(M, \omega)\right\},
$$

where $B^{2 n}(r)=\left\{\left.(x, y) \in \mathbb{R}^{2 n}|| x\right|^{2}+|y|^{2}<r^{2}\right\}$. It is the first nontrivial symplectic invariant. Denote by $Z^{2 n}(r)=\left\{(x, y) \in \mathbb{R}^{2 n} \mid x_{1}^{2}+y_{1}^{2}<r^{2}\right\}$.

\footnotetext{
${ }^{1}$ This paper is partially supported by the NNSF 10371007 and 10671017 of China and the Program for New Century Excellent Talents of the Education Ministry of China.

${ }^{2}$ Corresponding author
} 
Gromov [1] also proved

$$
\mathcal{W}_{G}\left(B^{2 n}(r), \omega_{0}\right)=\mathcal{W}_{G}\left(Z^{2 n}(r), \omega_{0}\right)=\pi r^{2}
$$

which implies the famous Gromov's nonsqueezing theorem.

Motivated by the properties of $\mathcal{W}_{G}$, Ekeland and Hofer [2] introduced the following notion. A functor $c$ from the category of all symplectic manifolds to $[0,+\infty]$ is called a symplectic capacity if it satisfies the following conditions. (monotonicity) If there is a symplectic embedding $\left(M_{1}, \omega_{1}\right) \rightarrow\left(M_{2}, \omega_{2}\right)$ of codimension zero then $c\left(M_{1}, \omega_{1}\right) \leq c\left(M_{2}, \omega_{2}\right)$;

(conformality) $c(M, \lambda \omega)=|\lambda| c(M, \omega)$ for every $\lambda \in \mathbb{R} \backslash\{0\}$; (nontriviality) $c\left(B^{2 n}(1), \omega_{0}\right)=\pi=c\left(Z^{2 n}(1), \omega_{0}\right)$.

By the monotonicity, $c$ is a symplectic invariant. It is easily checked that $\mathcal{W}_{G}$ is a symplectic capacity.

Hofer and Zehnder [5] introduced another important symplectic capacity $c_{H Z}$, called Hofer-Zehnder symplectic capacity. A smooth real function on a symplectic manifold $(M, \omega)$ is called admissible if there exist an nonempty open subset $U$ and a compact subset $K \subset M \backslash \partial M$ such that (a) $\left.H\right|_{U}=0$ and $\left.H\right|_{M \backslash K}=\max H$; (b) $0 \leq H \leq \max H$; (c) $\dot{x}=X_{H}(x)$ has no nonconstant periodic solutions of period less than 1. Here $X_{H}$ is defined by $\omega\left(X_{H}, v\right)=d H(v)$ for $v \in T M$. Denote by $\mathcal{H}_{a d}(M, \omega)$ the set of admissible Hamiltonians on $(M, \omega)$. The Hofer-Zehnder symplectic capacity of $(M, \omega)$ is defined by

$$
c_{H Z}(M, \omega)=\sup \left\{\max H \mid H \in \mathcal{H}_{a d}(M, \omega)\right\} .
$$

It and $\mathcal{W}_{G}$ always satisfy the following relation:

$$
\mathcal{W}_{G}(M, \omega) \leq c_{H Z}(M, \omega) \text {. }
$$

The symplectic capacities play important roles in the study of symplectic topology and Hamiltonian dynamics. However, their computations and estimations are difficult. In [6] the first author defined the concept of pseudo symplectic capacities, and specially constructed a pseudo symplectic capacity of Hofer-Zehnder type. Using the latter he computed Gromov symplectic width and Hofer-Zehnder symplectic capacity for many symplectic manifolds, see $[6,7,8]$. For more detailed study history of symplectic capacities the reader may refer to $[1,3,6]$ and references therein.

Recall that the classical domains of four types are

$$
\begin{aligned}
R_{I}(m, n) & =\left\{Z \in \mathbb{C}^{m \times n} \mid I^{(m)}-Z \bar{Z}^{\prime}>0\right\}, \\
R_{I I}(p) & =\left\{Z \in \mathbb{C}^{p \times p} \mid I^{(p)}-Z \bar{Z}^{\prime}>0, Z=Z^{\prime}\right\}, \\
R_{I I I}(q) & =\left\{Z \in \mathbb{C}^{q \times q} \mid I^{(q)}-Z \bar{Z}^{\prime}>0, Z=-Z^{\prime}\right\}, \\
R_{I V}(n) & =\left\{\left.Z \in \mathbb{C}^{n}|1+| Z Z^{\prime}\right|^{2}-2 Z \bar{Z}^{\prime}>0,1-\left|Z Z^{\prime}\right|^{2}>0\right\},
\end{aligned}
$$

see [9]. Here $Z \in \mathbb{C}^{m \times n}$ means that $Z$ is a complex $m \times n$ matrix, $Z^{\prime}$ is the transposed matrix of $Z$ and $H>0$ means that $H$ is a positive definite Hermitian matrix. 
Let $\omega_{0}^{(s)}$ denote the standard symplectic structure in $\mathbb{R}^{2 s}$. Identifying matric $Z=\left(z_{i j}\right)$ of order $m \times n$ with vector $z=\left(z_{11}, \cdots, z_{1 n}, z_{21}, \cdots, z_{2 n}, \cdots, z_{m 1}\right.$, $\left.\cdots, z_{m n}\right)$ in $\mathbb{C}^{m n}$, the standard symplectic form $\omega_{0}^{(m n)}$ on $\mathbb{C}^{m n}$ can be expressed as

$$
\omega_{0}^{(m n)}=\frac{\sqrt{-1}}{2} \operatorname{Tr}\left[d Z \wedge d \bar{Z}^{T}\right] .
$$

Using (1.2), it immediately follows from (7) and Theorem 1.15 in [6] that

$$
\mathcal{W}_{G}\left(R_{I}(m, n), \omega_{0}^{(m n)}\right)=c_{H Z}\left(R_{I}(m, n), \omega_{0}^{(m n)}\right)=\pi .
$$

Furthermore, by Lemma 4.1, Lemma 4.2 and (22) in [6], we immediately get

$$
\left\{\begin{array}{l}
\mathcal{W}_{G}\left(R_{I}\left(k_{1}, n_{1}\right) \times \cdots \times R_{I}\left(k_{r}, n_{r}\right), \omega_{0}^{\left(k_{1} n_{1}\right)} \oplus \cdots \oplus \omega_{0}^{\left(k_{r} n_{r}\right)}\right)=\pi \\
c_{H Z}\left(R_{I}\left(k_{1}, n_{1}\right) \times \cdots \times R_{I}\left(k_{r}, n_{r}\right), \omega_{0}^{\left(k_{1} n_{1}\right)} \oplus \cdots \oplus \omega_{0}^{\left(k_{r} n_{r}\right)}\right)=\pi
\end{array}\right.
$$

Note that $R_{I I}(p)$ is a symplectic submanifold of dimension $p(p+1)$ in $\left(\mathbb{C}^{p^{2}}, \omega_{0}^{\left(p^{2}\right)}\right)$. For $1 \leq i, j \leq p$, let $\eta_{i j}=1$ if $i=j$, and $\eta_{i j}=\sqrt{2}$ if $i<j$. Consider the following linear embedding

$$
\psi: R_{I I}(p) \rightarrow \mathbb{C}^{\frac{p(p+1)}{2}}, Z=\left(z_{i j}\right) \mapsto W=\left(\eta_{i j} z_{i j}\right)_{i \leq j},
$$

and denote by $\widetilde{R}_{I I}(p)$ the image of it. Then $\widetilde{R}_{I I}(p)$ is an open subset in $\mathbb{R}^{p(p+1)}$, and $\psi$ is a symplectomorphism from $\left(R_{I I}(p),\left.\omega_{0}^{\left(p^{2}\right)}\right|_{R_{I I}(p)}\right)$ to $\left(\widetilde{R}_{I I}(p), \omega_{0}^{\left(\frac{p(p+1)}{2}\right)}\right)$.

Similarly, $R_{I I I}(q)$ is a symplectic submanifold of dimension $q(q-1)$ in $\left(\mathbb{C}^{q^{2}}, \omega_{0}^{\left(q^{2}\right)}\right)$, and the linear map

$$
\varphi: R_{I I I}(q) \rightarrow \mathbb{C}^{\frac{q(q-1)}{2}}, Z=\left(z_{i j}\right) \mapsto W=\left(\sqrt{2} z_{i j}\right)_{i<j}
$$

is a symplectic embedding of codimension zero into $\left(\mathbb{R}^{q(q-1)}, \omega_{0}^{\left(\frac{q(q-1)}{2}\right)}\right)$. Let $\widetilde{R}_{I I I}(q)$ denote the image of $\varphi$.

Theorem 1.1. For classical domains of other three types the following estimations hold.

$$
\begin{aligned}
& \mathcal{W}_{G}\left(R_{I I}(p),\left.\omega_{0}^{\left(p^{2}\right)}\right|_{R_{I I}(p)}\right)=c_{H Z}\left(R_{I I}(p),\left.\omega_{0}^{\left(p^{2}\right)}\right|_{R_{I I}(p)}\right)=\pi, \\
& \pi \leq \mathcal{W}_{G}\left(R_{I I I}(q),\left.\omega_{0}^{\left(q^{2}\right)}\right|_{R_{I I I}(q)}\right) \leq c_{H Z}\left(R_{I I I}(q),\left.\omega_{0}^{\left(q^{2}\right)}\right|_{R_{I I I}(q)}\right) \leq 2 \pi, \\
& \frac{\pi}{2} \leq \mathcal{W}_{G}\left(R_{I V}(n), \omega_{0}^{(n)}\right) \leq c_{H Z}\left(R_{I V}(n), \omega_{0}^{(n)}\right) \leq \pi
\end{aligned}
$$

Remark 1.2. (i) Taking $q=2$, the map in (1.6) gives a symplectomorphism from $\left(R_{I I I}(2),\left.\omega_{0}^{(4)}\right|_{R_{I I I}(2)}\right)$ to $\left(B^{2}(\sqrt{2}), \omega_{0}^{(1)}\right)$. Hence $\mathcal{W}_{G}\left(R_{I I I}(2),\left.\omega_{0}^{(4)}\right|_{R_{I I I}(2)}\right)=$ $c_{H Z}\left(R_{I I I}(2),\left.\omega_{0}^{(4)}\right|_{R_{I I I}(2)}\right)=2 \pi$. This shows that the upper bound in (1.8) is optimal.

(ii) Take $n=1$, then $R_{I V}(1)=B^{2}(1)$ and thus $\mathcal{W}_{G}\left(R_{I V}(1), \omega_{0}\right)=$ $c_{H Z}\left(R_{I V}(1), \omega_{0}\right)=\pi$ because $\mathcal{W}_{G}$ and $c_{H Z}$ are symplectic capacities. This 
shows that the upper bound in (1.9) is optimal. To show the optimality of the lower bound in (1.9), consider the linear map given by Lemma 2.1.3 in [9],

$$
\psi: R_{I V}(4) \rightarrow R_{I}(2,2),\left(z_{1}, z_{2}, z_{3}, z_{4}\right) \mapsto W=\left(\begin{array}{cc}
w_{1} & w_{3} \\
w_{4} & w_{2}
\end{array}\right),
$$

where $w_{1}=z_{1}+i z_{2}, w_{2}=z_{1}-i z_{2}, w_{3}=i z_{3}-z_{4}$ and $w_{4}=i z_{3}+z_{4}$. It is directly computed that

$$
\begin{aligned}
\psi^{*} \omega_{0}^{(4)} & =\frac{\sqrt{-1}}{2}\left(d w_{1} \wedge d \bar{w}_{1}+d w_{2} \wedge d \bar{w}_{2}+d w_{3} \wedge d \bar{w}_{3}+d w_{4} \wedge d \bar{w}_{4}\right) \\
& =\sqrt{-1}\left(d z_{1} \wedge d \bar{z}_{1}+d z_{2} \wedge d \bar{z}_{2}+d z_{3} \wedge d \bar{z}_{3}+d z_{4} \wedge d \bar{z}_{4}\right)=2 \omega_{0}^{(4)} .
\end{aligned}
$$

Then from the conformality of $\mathcal{W}_{G}$ and (1.3) it follows that $\mathcal{W}_{G}\left(R_{I V}(4), \omega_{0}^{(4)}\right)=$ $\pi / 2$.

\section{Proofs of Theorem 1.1}

The following three lemmas can be found on pages 349-353 of [9].

Lemma 2.1. For any $Z \in \mathbb{C}^{m \times n}$ with $m \leq n$, there exist unitary matrices $U$ of order $m$ and $V$ of order $n$ such that $Z=U\left(\operatorname{diag}\left(\lambda_{1}, \cdots, \lambda_{m}\right), O\right) V$ for some $\lambda_{1} \geq \cdots \lambda_{m} \geq 0$, where $\operatorname{diag}\left(\lambda_{1}, \cdots, \lambda_{m}\right)$ denotes the diagonal matrix of order $m$, and $O$ is the $m \times(n-m)$ zero matrix.

Consequently, for a given $Z \in \mathbb{C}^{m \times n}$, it holds that $I^{(m)}-Z \bar{Z}^{T}>0$ if and only if $I^{(n)}-\bar{Z}^{T} Z>0$.

Lemma 2.2. For any symmetric matrix $Z \in \mathbb{C}^{p \times p}$, there exist a unitary matric $U \in \mathbf{U}(p)$ and real numbers $\lambda_{1} \geq \lambda_{2} \geq \ldots \lambda_{p} \geq 0$, such that $Z=$ $U^{T} \operatorname{diag}\left(\lambda_{1}, \cdots, \lambda_{p}\right) U$.

Lemma 2.3. For any anti-symmetric matrix $Z \in \mathbb{C}^{q \times q}$, there exists a unitary matric $U \in \mathbf{U}(q)$ such that

$$
\begin{aligned}
& Z=U\left[\left(\begin{array}{ll}
0 & \lambda_{1} \\
-\lambda_{1} & 0
\end{array}\right) \dot{+} \cdot \dot{+}\left(\begin{array}{ll}
0 & \lambda_{\gamma} \\
-\lambda_{\gamma} & 0
\end{array}\right)\right] \bar{U}^{T} \quad \text { as } \quad q=2 \gamma \\
& Z=U\left[\left(\begin{array}{ll}
0 & \lambda_{1} \\
-\lambda_{1} & 0
\end{array}\right) \dot{+} \cdot \dot{+}\left(\begin{array}{ll}
0 & \lambda_{\gamma} \\
-\lambda_{\gamma} & 0
\end{array}\right) \dot{+} 0\right] \bar{U}^{T} \quad \text { as } \quad q=2 \gamma+1 .
\end{aligned}
$$

Here the direct sum " $M \dot{+} N$ " of matries $M$ and $N$ is defined by $\left(\begin{array}{ll}M & 0 \\ 0 & N\end{array}\right)$.

By the proof of Theorem 4.1.4 on page 165 of [9] we have

Lemma 2.4. The ball $B^{2 m n}(1)$ is an open subset of $R_{I}(m, n)$. 
Lemma 2.5. $R_{I}(m, n) \subseteq Z^{2 m n}(1)$.

Proof. For $Z \in R_{I}(m, n)$, let $A=I^{(m)}-Z_{Z^{T}}$. Then $A=\left(a_{i j}\right)>0$, and thus $a_{11}=1-\sum_{i=1}^{n}\left(z_{1 i} \bar{z}_{1 i}\right)=1-\sum_{i=1}^{n}\left|z_{1 i}\right|^{2}>0$. In particular, $\left|z_{11}\right|<1$, that is, $Z \in Z^{2 m n}(1)$.

It immediately follows from the monotonicity of $c_{H Z}$ that $c_{H Z}\left(R_{I}(m, n), \omega^{(m, n)}\right)$ $\leq \pi$, which was first proved by the first author with his pseudo symplectic capacity theory in [6]. Similarly, it is also easy to see that Lemma 2.4 and Lemma 2.5 may give a simple proof of (1.4).

Proof of (1.7). By the arguments above Theorem 1.1, we have

$$
\begin{aligned}
& \mathcal{W}_{G}\left(R_{I I}(p),\left.\omega_{0}^{\left(p^{2}\right)}\right|_{R_{I I}(p)}\right)=\mathcal{W}_{G}\left(\widetilde{R}_{I I}(p), \omega_{0}^{\left(\frac{p(p+1)}{2}\right)}\right) \quad \text { and } \\
& c_{H Z}\left(R_{I I}(p),\left.\omega_{0}^{\left(p^{2}\right)}\right|_{R_{I I}(p)}\right)=c_{H Z}\left(\widetilde{R}_{I I}(p), \omega_{0}^{\left(\frac{p(p+1)}{2}\right)}\right) .
\end{aligned}
$$

For $Z \in R_{I I}(p)$, let $W=\psi(Z)=\left(w_{i j}\right)_{i \leq j}$. As in the proof of Lemma 2.5 above we can show that $\left|z_{11}\right|<1$. So $\left|w_{11}\right|=\left|z_{11}\right|<1$. It follows that $\widetilde{R}_{I I}(p)=\psi\left(R_{I I}(p)\right) \subset Z^{p(p+1)}(1)$. Therefore (1.1) and (1.2) give

$$
\mathcal{W}_{G}\left(\widetilde{R}_{I I}(p), \omega_{0}^{\left(\frac{p(p+1)}{2}\right)}\right) \leq c_{H Z}\left(\widetilde{R}_{I I}(p), \omega_{0}^{\left(\frac{p(p+1)}{2}\right)}\right) \leq \pi
$$

For a symmetric matrix $Z \in \mathbb{C}^{p \times p}$, by Lemma 2.2, there exist a unitary matric $U \in \mathbf{U}(p)$ and $\lambda_{1} \geq \lambda_{2} \geq \ldots \lambda_{p} \geq 0$, such that $Z=U^{T} \operatorname{diag}\left(\lambda_{1}, \cdots, \lambda_{p}\right) U$ and thus

$$
I^{(p)}-Z \bar{Z}^{T}=I^{(p)}-U^{T} \operatorname{diag}\left(\lambda_{1}^{2}, \cdots, \lambda_{p}^{2}\right) \bar{U}=U^{T} \operatorname{diag}\left(1-\lambda_{1}^{2}, \cdots, 1-\lambda_{p}^{2} \bar{U} .\right.
$$

It follows that $Z \in R_{I I}(p) \Leftrightarrow I^{(p)}-Z_{\bar{Z}}^{T}>0 \Leftrightarrow \lambda_{i}<1(i=1, \cdots, p)$. For $\widetilde{Z}=\left(\tilde{z}_{i j}\right)_{1 \leq i \leq j \leq p} \in B^{p(p+1)}(1)$, we define a symmetric matrix $Z=\left(z_{i j}\right) \in \mathbb{C}^{p \times p}$ as follows: $z_{i i}=\tilde{z}_{i i}$ for $i=1, \cdots, p, z_{i j}=\tilde{z}_{i j} / \sqrt{2}$ for $i<j$, and $z_{i j}=\tilde{z}_{j i} / \sqrt{2}$ for $i>j$. As above we have an expression $Z=U^{T} \operatorname{diag}\left(\lambda_{1}, \cdots, \lambda_{p}\right) U$. Then

$$
1>\|\widetilde{Z}\|^{2}=\sum_{1 \leq i \leq j \leq p}\left|\tilde{z}_{i j}\right|^{2}=\sum_{1 \leq i, j \leq p}\left|z_{i j}\right|^{2}=\operatorname{Tr}\left(Z \bar{Z}^{T}\right)=\sum_{i=1}^{p} \lambda_{i}^{2},
$$

Hence $Z \in R_{I I}(p)$ and $\widetilde{Z}=\psi(Z) \in \widetilde{R}_{I I}(p)$. It follows that $B^{p(p+1)}(1) \subseteq \widetilde{R}_{I I}(p)$ and $\mathcal{W}_{G}\left(\widetilde{R}_{I I}(p), \omega_{0}^{\left(\frac{p(p+1)}{2}\right)}\right) \geq \pi$. This and (2.1) together give (1.7).

Proof of (1.8). The proof is similar to that of (1.7). We still give it for clearness. By (1.6), we have

$$
\begin{aligned}
& \mathcal{W}_{G}\left(R_{I I I}(q),\left.\omega_{0}^{\left(q^{2}\right)}\right|_{R_{I I I}(q)}\right)=\mathcal{W}_{G}\left(\widetilde{R}_{I I I}(q), \omega_{0}^{\left(\frac{q(q-1)}{2}\right)}\right) \quad \text { and } \\
& c_{H Z}\left(R_{I I I}(q),\left.\omega_{0}^{\left(q^{2}\right)}\right|_{R_{I I I}(q)}\right)=c_{H Z}\left(\widetilde{R}_{I I I}(q), \omega_{0}^{\left(\frac{q(q-1)}{2}\right)}\right) .
\end{aligned}
$$

For $Z \in R_{I I I}(q)$, as in the proof of Lemma 2.5 we get that $a_{11}=1-$ $\sum_{i=1}^{n}\left(z_{1 i} \bar{z}_{1 i}\right)=1-\sum_{i=1}^{n}\left|z_{1 i}\right|^{2}>0$. It implies that $\left|z_{12}\right|<1$. Let $W=$ 
$\varphi(Z)=\left(w_{i j}\right)_{i<j}$. Then $\left|w_{12}\right|<\sqrt{2}$, and thus $\widetilde{R}_{I I I}(q) \subseteq Z^{q(q-1)}(\sqrt{2})$. Hence

$$
\mathcal{W}_{G}\left(\widetilde{R}_{I I I}(q), \omega_{0}^{\left(\frac{q(q-1)}{2}\right)}\right) \leq c_{H Z}\left(\widetilde{R}_{I I I}(q), \omega_{0}^{\left(\frac{q(q-1)}{2}\right)}\right) \leq 2 \pi .
$$

On the other hand, for an anti-symmetric matrix $Z \in \mathbb{C}^{q \times q}$, when $q=2 \gamma$, by Lemma 2.3 we have a unitary matric $U \in \mathbf{U}(q)$ and $\lambda_{1} \geq \lambda_{2} \geq \ldots \lambda_{\gamma} \geq 0$, such that

$$
Z=U\left[\left(\begin{array}{ll}
0 & \lambda_{1} \\
-\lambda_{1} & 0
\end{array}\right) \dot{+} \cdots \dot{+}\left(\begin{array}{ll}
0 & \lambda_{\gamma} \\
-\lambda_{\gamma} & 0
\end{array}\right)\right] \bar{U}^{T} \text { and thus }
$$

$I^{(q)}-Z \bar{Z}^{T}=U \operatorname{diag}\left[\left(\begin{array}{ll}1-\lambda_{1}^{2} & 0 \\ 0 & 1-\lambda_{1}^{2}\end{array}\right) \dot{+} \cdot \cdots \dot{+}\left(\begin{array}{ll}1-\lambda_{\gamma}^{2} & 0 \\ 0 & 1-\lambda_{\gamma}^{2}\end{array}\right)\right] \bar{U}^{T}$.

Similarly, when $q=2 \gamma+1$, we have a unitary matric $U \in \mathbf{U}(q)$ and $\lambda_{1} \geq \lambda_{2} \geq$ $\ldots \lambda_{\gamma} \geq 0$, such that

$$
Z=U \operatorname{diag}\left[\left(\begin{array}{ll}
0 & \lambda_{1} \\
-\lambda_{1} & 0
\end{array}\right) \dot{+} \cdots \dot{+}\left(\begin{array}{ll}
0 & \lambda_{\gamma} \\
-\lambda_{\gamma} & 0
\end{array}\right) \dot{+} 0\right] \bar{U}^{T} \text { and }
$$

$$
I^{(q)}-Z \bar{Z}^{T}=U \operatorname{diag}\left[\left(\begin{array}{ll}
1-\lambda_{1}^{2} & 0 \\
0 & 1-\lambda_{1}^{2}
\end{array}\right) \dot{+} \cdots \dot{+}\left(\begin{array}{ll}
1-\lambda_{\gamma}^{2} & 0 \\
0 & 1-\lambda_{\gamma}^{2}
\end{array}\right) \dot{+} 1\right] \bar{U}^{T} .
$$

It follows that $Z \in R_{I I I}(q) \Leftrightarrow I^{(q)}-Z \bar{Z}^{T}>0 \Leftrightarrow \lambda_{i}<1(i=1, \cdots, \gamma)$.

For $\widetilde{Z}=\left(\tilde{z}_{i j}\right)_{1 \leq i<j \leq p} \in B^{q(q-1)}(1)$, we define an anti-symmetric matrix $Z=$ $\left(z_{i j}\right) \in \mathbb{C}^{q \times q}$ by setting $z_{i j}=\tilde{z}_{i j} / \sqrt{2}$ for $i<j$, and $z_{i j}=-\tilde{z}_{j i} / \sqrt{2}$ for $i>j$. As above, using (2.3) and (2.4) for this $Z$ we get

$$
1>\|\widetilde{Z}\|^{2}=\sum_{1 \leq i<j \leq q}\left|\tilde{z}_{i j}\right|^{2}=\sum_{1 \leq i, j \leq q}\left|z_{i j}\right|^{2}=\operatorname{Tr}\left(Z \bar{Z}^{T}\right)=\sum_{i=1}^{\gamma} \lambda_{i}^{2}
$$

Hence $Z \in R_{I I I}(q), \widetilde{Z}=\varphi(Z) \in \widetilde{R}_{I I I}(q)$ and $B^{q(q-1)}(1) \subseteq \widetilde{R}_{I I I}(q)$. It follows that $\mathcal{W}_{G}\left(\widetilde{R}_{I I I}(q), \omega_{0}^{\left(\frac{q(q-1)}{2}\right)}\right) \geq \pi$. This and (2.2) together lead to (1.8).

Proof of (1.9). When $n=1$ we have proved the claims in Remark 1.2. When $n \geq 2$, the desired estimates are easily obtained from the arguments on pages 169-170 of [9]. In fact, it is easy to prove that the linear map $\phi: \mathbb{C}^{n} \rightarrow \mathbb{C}^{n}$ given by

$$
w_{1}=z_{1}+i z_{2}, w_{2}=z_{1}-i z_{2}, w_{k}=\sqrt{2} z_{k}, k=3, \cdots, n,
$$


satisfies $\phi^{*} \omega_{0}^{(n)}=2 \omega_{0}^{(n)}$. Denote by $\widetilde{R}_{I V}(n)=\phi\left(R_{I V}(n)\right)$. The conformality of symplectic capabilities implies

$$
\begin{aligned}
\mathcal{W}_{G}\left(\widetilde{R}_{I V}(n), \omega_{0}^{(n)}\right) & =\mathcal{W}_{G}\left(R_{I V}(n), \phi^{*} \omega_{0}^{(n)}\right) \\
& =\mathcal{W}_{G}\left(R_{I V}(n), 2 \omega_{0}^{(n)}\right)=2 \mathcal{W}_{G}\left(R_{I V}(n), \omega_{0}^{(n)}\right), \\
c_{H Z}\left(\widetilde{R}_{I V}(n), \omega_{0}^{(n)}\right) & =c_{H Z}\left(R_{I V}(n), \phi^{*} \omega_{0}^{(n)}\right) \\
& =c_{H Z}\left(R_{I V}(n), 2 \omega_{0}^{(n)}\right)=2 c_{H Z}\left(R_{I V}(n), \omega_{0}^{(n)}\right) .
\end{aligned}
$$

On the other hand it was proved on page 170 of [9] that $B^{2 n}(1) \subseteq \widetilde{R}_{I V}(n) \subseteq$ $B^{2 n}(\sqrt{2})$. Hence

$$
\pi \leq \mathcal{W}_{G}\left(\widetilde{R}_{I V}(n), \omega_{0}^{(n)}\right) \leq c_{H Z}\left(\widetilde{R}_{I V}(n), \omega_{0}^{(n)}\right) \leq 2 \pi
$$

The desired (1.9) follows.

\section{REFERENCES}

[1] K. Cieliebak, H. Hofer, J. Latschev, F. Schlenk, Quantitative symplectic geometry. Preprint, math. SG/0506191.

[2] I. Ekeland, and H. Hofer, Symplectic topology and Hamiltonian dynamics, Mathematische Zeitschrift, 200(1989), 355-378.

[3] V. L. Ginzburg, The Weinstein conjecture and the theorems of nearby and almost existence. The breadth of symplectic and Poisson geometry, 139-172, Progr. Math., 232, Birkhüser Boston, Boston, MA, 2005.

[4] M. Gromov, Pseudoholomorphic curves in symplectic manifolds, Invent. Math., 82 (1985), 307-347.

[5] H. Hofer and E. Zehnder, A new capacity for symplectic manifolds, In Analysis et cetera (ed. P. Rabinowitz and E. Zehnder), pp. 405-429. Academic Press, New York, 1990.

[6] G. C. Lu, Gromov-Witten invariants and pseudo symplectic capacities, Israel Journal of Mathematics 156(2006), 1-63.

[7] G. C. Lu, Symplectic capacities of toric manifolds and related results, Nagoya mathematical Journal, 181(2006), 149-184.

[8] G. C. Lu, Finiteness of the Hofer-Zehnder capacity of neighborhoods of symplectic submanifolds, International Mathematics Research Notices, Volume 2006, no.8, Art. ID 76520, 1-33.

[9] Q. K. Lu, Classicial Manifolds and Classicial Domains(in Chinese) Shanghai: Shanghai Science and Tech. Publ., Shanghai, 1963.

\section{Received: October 18, 2006}

